\title{
EGFR Mutation Analysis of Circulating Tumor DNA Using an Improved PNA-LNA PCR Clamp Method
}

\author{
Kana Watanabe, ${ }^{1}$ Tatsuro Fukuhara, ${ }^{1}$ Yoko Tsukita, ${ }^{1}$ Mami Morita, ${ }^{1}$ Aya Suzuki, \\ Nobuyuki Tanaka, ${ }^{2}$ Hiroshi Terasaki, ${ }^{3}$ Toshihiro Nukiwa, ${ }^{4}$ and Makoto Maemondo ${ }^{1}$ \\ ${ }^{1}$ Department of Respiratory Medicine, Miyagi Cancer Center, 47-1 Nodayama, Medeshima-Shiote, Natori 981-1293, Japan \\ ${ }^{2}$ Division of Cancer Biology and Therapeutics, Miyagi Cancer Center Research Institute, Natori, Japan \\ ${ }^{3}$ Molecular Genetic Research Department, LSI Medience Corporation, Tokyo, Japan \\ ${ }^{4} J a p a n$ Anti-Tuberculosis Association, Tokyo, Japan
}

Correspondence should be addressed to Makoto Maemondo; maemondo-ma693@aioros.ocn.ne.jp

Received 15 May 2016; Revised 10 June 2016; Accepted 15 June 2016

Academic Editor: Elisa Giovannetti

Copyright (C) 2016 Kana Watanabe et al. This is an open access article distributed under the Creative Commons Attribution License, which permits unrestricted use, distribution, and reproduction in any medium, provided the original work is properly cited.

\begin{abstract}
Introduction. Rebiopsies have become more crucial in non-small cell lung cancer (NSCLC). Instead of invasive biopsies, development of collecting biological data of the tumor from blood samples is expected. We conducted a prospective study to assess the feasibility of detection of epidermal growth factor receptor (EGFR) mutation in plasma samples. Method. NSCLC patients harboring EGFR activating mutations, who were going to receive EGFR-tyrosine kinase inhibitors (TKIs) as first-line treatment, were enrolled in this study. Plasma EGFR activating mutations and the T790M resistance mutation were analyzed by an improved PNA-LNA PCR clamp method, characterized by a 10 -fold or more sensitivity compared with the original methods. Result. Six patients with wild-type EGFR and 24 patients with EGFR mutations were enrolled in this study. Pretreatment plasma samples achieved sensitivity of $79 \%$. The 6 patients with wild-type EGFR were all negative for plasma EGFR mutations. At the time of disease progression, plasma T790M mutation was detected in 8 of 16 cases. Absence of T790M before and during TKI treatment and disappearance of activating mutations during TKI treatment were considered as predictors of EGFR-TKIs efficacy. Conclusion. We were able to detect EGFR mutations in plasma samples by using an improved PNA-LNA PCR clamp method.
\end{abstract}

\section{Introduction}

EGFR mutations have become crucial predictors of the effect of epidermal growth factor receptor-tyrosine kinase inhibitors (EGFR-TKIs) in non-small cell lung cancer (NSCLC). Several reports demonstrated that gefitinib, erlotinib, and afatinib were significantly superior to chemotherapy in terms of efficacy [1-6]. First-line EGFR-TKIs have become standard treatments in NSCLC harboring EGFR mutations, substituting platinum doublet. Detection of driver mutations including EGFR mutations and anaplastic lymphoma kinase rearrangements has become a routine test at diagnosis as well as distinction of the histological type. One clinical issue is how to precisely detect driver mutations. Detection rate is influenced by various factors including size, fixation, preservation of samples, and extraction of DNA. Another issue related to EGFR mutation analysis is how to detect resistant EGFR mutations. First generation TKIs, gefitinib and erlotinib, cannot control disease progression for more than a year in numerous patients with EGFR mutations. More than $50 \%$ of mechanisms of resistance are due to the T790M mutation [7]. So far, detection of the T790M mutation availed just for negative selection. Recently, 3rd generation TKIs, effective on T790M mutated tumors, were developed and have become available in the United States. Consequently, it will be essential to confirm the existence of T790M mutations in tumor resistant to EGFR-TKIs. Though rebiopsies have been recommended to confirm EGFR mutation status, it is not always possible to obtain them because of the tumor size and the sites of primary or metastatic tumors. Therefore, less harmful and convenient procedures were desired.

A new approach to detect mutations in circulating tumor DNA (ctDNA) from plasma, so-called liquid biopsy, is being developed. Though liquid biopsies include ctDNA, circulating tumor cells, and exosomal RNA, the term liquid biopsy (liquid biopsy refers to collection of circulating tumor DNA 
in plasma in this report) was used referring to ctDNA in this study. Blood specimens are easy to obtain compared with cytological and histological samples, but previous reports have shown that detection rates of mutations in liquid biopsies were too low for clinical use [8]. There are several methods to detect plasma cell-free DNA, including highsensitive PCR methods, digital PCR (dPCR), and nextgeneration sequencing (NGS). We have used a high-sensitive PCR method, PNA-LNA PCR clamp analysis, established by our group [9]. We have conducted several clinical studies for EGFR-mutated NSCLCs detected with this PNA-LNA clamp method [1, 10-12]. Since the original PNA-LNA PCR clamp method does not have high sensitivity for liquid biopsy, we developed an improved PNA-LNA PCR clamp method with higher detection rate which has become an appropriate method for liquid biopsy. We conducted a prospective study on the effect of first-generation EGFR-TKIs on EGFR mutations using the highly sensitive PNA-LNA PCR clamp analysis of plasma samples.

\section{Methods}

2.1. Patients. The prospective study was conducted to analyze EGFR mutations in plasma samples. We enrolled inoperable stage III or stage IV NSCLC patients harboring EGFR activating mutations who were supposed to receive EGFR-TKIs as first-line treatment. Other eligible criteria were the following: activating EGFR mutations (exon 19 deletion, L858R, and minor mutations) detected by PNA-LNA PCR clamp method, Eastern Cooperative Oncology Group (ECOG) performance status (PS) 0 to 2, and adequate organ function. Written informed consent for the use of blood samples was obtained from all patients. The study protocol was approved by the Ethical Review Committee of the Miyagi Cancer Center.

\subsection{Plasma Sample Collection and EGFR Mutation Analysis.} Whole blood samples $(14 \mathrm{~mL})$ were collected in ethylenediaminetetraacetic acid (EDTA) tubes before TKI treatment (P0), 2 months after the initiation of the treatment (P1), and after disease progression (P2). Samples were mixed thoroughly, and plasma isolated by centrifugation at $2000 \times \mathrm{g}$ for $10 \mathrm{~min}$ was stored at $-20^{\circ} \mathrm{C}$. Collection of cytohistological samples was mandatory before treatment and was recommended after progression of disease (PD). DNA was extracted from plasma samples with QIAamp Circulating Nucleic Acid or from cytohistological samples with QIAamp DNA FFPE Tissue (QIAGEN, Hilden, Germany). PCR primers were designed to amplify G719X, exon 19 deletion, T790M, L858R, and L861Q. Here, we briefly explain mechanism of PNA-LNA clamp method (supplemental figure in Supplementary Material available online at http://dx.doi.org/10.1155/2016/5297329). This system utilizes PNA/LNA that binds complementary bases more firmly compared to DNA/DNA binding. PNA clamps were composed to be complementary to the respective wild-type alleles and LNA probes are constructed complementary to each mutation allele (Supplemental Table 1). Namely, PNA clamp hinders amplification of wild-type alleles and mutation sequences are effectively detected by LNA probe in real-time PCR cycling.

Samples were collected and stored at the Miyagi Cancer Center, and mutations were analyzed in the central laboratory, LSI Medience Corporation (Tokyo, Japan). PCR reaction conditions are shown in Supplemental Table 2. This improved PNA-LNA clamp method achieved less than $0.1 \%$ detection rate by using smaller PCR products and by increasing the number of cycles from 45 to 50 using a LightCycler 480 Instrument (Roche). Wild-type EGFR samples were also included in the mutation positive samples to determine the specificity of these analyses. Data of mutations obtained from cytohistological samples were blinded to investigators in the central laboratory to avoid biases.

2.3. Endpoints. We prospectively evaluated concordance rate of EGFR mutations between plasma samples and cytohistological samples using the improved PNA-LNA PCR clamp method and tried to determine the factors influencing detection rate of EGFR mutations in the plasma. The relation between the effect of EGFR-TKI and the status of EGFR activating mutations and T790M mutation in blood samples before and during EGFR-TKI treatment was also analyzed. This study focuses not only on T790M mutation but also on activating mutations; alternation of activating or resistance mutations during TKI treatment was assessed as a predictive factor of TKI treatment efficacy.

2.4. Statistical Analysis. The chi-square test was used to assess detection rate of EGFR mutations in the plasma among characteristics including intrathoracic or extrathoracic lesions. $P$ values were two-tailed, and $P<0.01$ was considered statistically significant. Progression-free survival (PFS) was defined as the time from the starting day of EGFR-TKI treatment. PFS and overall survival (OS) were calculated using the Kaplan-Meier method, and correlation between EGFR mutation status and effects of TKIs was performed by log-rank test. All the analyses were conducted using SPSS version 12 (IBM SPSS Statistics, IBM, Tokyo, Japan).

\section{Results}

3.1. Patients' Characteristics. A total of 24 lung adenocarcinoma patients met the enrollment criteria and participated in the study from December 2012 to March 2014. This study was performed at the Miyagi Cancer Center. Patients' characteristics are shown in Table 1 . In summary, the median age was 67.0 years, 16 patients $(66.7 \%)$ were female, 18 patients (75.0\%) were never smokers, 22 patients $(91.7 \%)$ had ECOG PS 0-1, and 22 patients ( $91.7 \%$ ) presented with stage IV disease (8 patients with Mla and 14 patients with Mlb metastases).

3.2. Correlation of EGFR Mutation Status between Tumor and Plasma Samples. The EGFR mutation status in tumors was initially analyzed by the conventional PNA-LNA PCR clamp method and the results are shown in Table 1 . In 24 patients with EGFR mutations, 15 patients had exon 19 deletions, 8 patients had exon 21 L858R mutation, and 1 patient had exon 
TABLE 1: Patients' characteristics.

\begin{tabular}{|c|c|c|c|c|}
\hline & \multirow{2}{*}{ EGFR mutant (biopsy sample) } & \multicolumn{2}{|c|}{ EGFR mutant (biopsy sample) } & \multirow{2}{*}{ Wild-type EGFR (biopsy sample) } \\
\hline & & Plasma EGFR mut+ & Plasma EGFR mut- & \\
\hline Total, $n$ & 24 & 19 & 5 & 6 \\
\hline \multicolumn{5}{|l|}{ Age } \\
\hline Median (range) & $67(46-87)$ & $67(46-87)$ & $68(55-84)$ & $70(64-79)$ \\
\hline \multicolumn{5}{|l|}{ Gender } \\
\hline Female & $16(66.7)$ & $11(57.9)$ & $5(100)$ & $1(16.7)$ \\
\hline Male & $8(33.3)$ & $8(42.1)$ & $0(0.0)$ & $5(83.3)$ \\
\hline \multicolumn{5}{|l|}{ Smoking status } \\
\hline Never & $18(75 \%)$ & $14(73.7)$ & $4(80.0)$ & $2(33.3)$ \\
\hline Former & $3(12.5)$ & $2(10.5)$ & $1(20.0)$ & $1(16.7)$ \\
\hline Current & $3(12.5)$ & $3(15.8)$ & $0(0.0)$ & $3(50.0)$ \\
\hline \multicolumn{5}{|l|}{ ECOG PS } \\
\hline 0 & $8(33.3)$ & $5(26.3)$ & $3(60.0)$ & $1(16.7)$ \\
\hline 1 & $14(58.3)$ & $12(63.2)$ & $2(40.0)$ & $5(83.3)$ \\
\hline 2 & $2(8.3)$ & $2(10.5)$ & $0(0.0)$ & $0(0.0)$ \\
\hline \multicolumn{5}{|l|}{ Stage } \\
\hline IIA & $0(0.0)$ & $0(0.0)$ & $0(0.0)$ & $1(16.7)$ \\
\hline IIIA & $2(8.3)$ & $1(5.3)$ & $1(20.0)$ & $1(16.7)$ \\
\hline IV & $22(91.7)$ & $18(94.7)$ & $4(80.0)$ & $4(66.7)$ \\
\hline IV-M1a & $8(36.4)$ & $4(22.2)$ & $4(100)$ & $1(16.7)$ \\
\hline IV-M1b & $14(63.6)$ & $14(77.8)$ & $0(0.0)$ & $3(50.0)$ \\
\hline \multicolumn{5}{|c|}{ Tumor EGFR mutation status } \\
\hline Wild type & 0 & 0 & 0 & $6(100)$ \\
\hline del19 & $15(62.5)$ & $12(63.2)$ & $3(60.0)$ & 0 \\
\hline L858R & $8(33.3)$ & $7(36.8)$ & $1(20.0)$ & 0 \\
\hline L861Q & $1(4.2)$ & $0(0.0)$ & $1(20.0)$ & 0 \\
\hline
\end{tabular}

TABLE 2: Correlation of EGFR mutation status between tissue and plasma samples before EGFR-TKI treatment.

\begin{tabular}{lccc}
\hline & \multicolumn{2}{c}{ Pretreatment plasma: P0 } & Total \\
& Positive & Negative & \\
\hline Pretreatment tissue & & & \\
$\quad$ Positive & 19 & 5 & 24 \\
$\quad$ Negative & 0 & 6 & 6 \\
\hline Total & 19 & 11 & 30 \\
\hline
\end{tabular}

Sensitivity, $79.2 \%$; specificity, $100 \%$.

18 L861Q mutation. Furthermore, 6 patients with cytohistological wild-type EGFR were analyzed for plasma EGFR to evaluate the specificity of plasma EGFR mutation status. The results of the correlation of EGFR mutation status between tumor and baseline plasma samples are shown in Table 2. Of the 24 patients with EGFR-mutant tumors, 19 patients had detectable EGFR mutations in baseline plasma specimens with sensitivity of $79.2 \%$ and sensitivity for common mutations (exon 19 deletion and L858R mutation) of $82.4 \%$. All mutation types in plasma-positive patients were consistent between plasma and tumor specimens. Of 6 patients with wild-type EGFR tumors, the plasma samples were all negative for EGFR mutations. Therefore, the specificity was $100 \%$. As shown in Table 1, some factors including uncommon mutations and distal metastases might be correlated with the detection rate. Patients without extrathoracic metastases showed a significantly lower detection rate of plasma EGFR mutation than those with extrathoracic metastases $(5 / 10$ versus $14 / 14 ; P<0.003)$. There was no significant difference between the detection rate of exon 19 deletion and L858R mutation.

3.3. Efficacy of EGFR-TKI Treatment In Patients with EGFRMutant Tumors. All 24 patients with EGFR-mutant tumors received EGFR-TKI as first-line treatment: 23 received gefitinib and 1 received erlotinib. Twenty-three patients achieved partial response $(\mathrm{PR})$ and 1 patient had stable disease. No patient achieved complete response. The objective response rate (ORR) and disease control rate (DCR) were $95.8 \%$ and $100 \%$, respectively. There was no significant difference in ORR or DCR between patients with positive and negative baseline plasma EGFR mutations (94.7\% versus $100 \%$ and $100 \%$ versus $100 \%$, resp.). We evaluated the effect of treatment duration of TKIs, in addition to tumor response. Five cases negative for plasma EGFR mutations at baseline (P0) were still negative 2 months after initiation of the treatment (P1) (group A). Of 19 cases positive for plasma activating EGFR mutations at P0, 12 cases had no plasma mutations at P1 (group B) and 6 cases were still positive at P1 (group C) (Figure 1). In group $\mathrm{C}$, the detected activating mutations were 

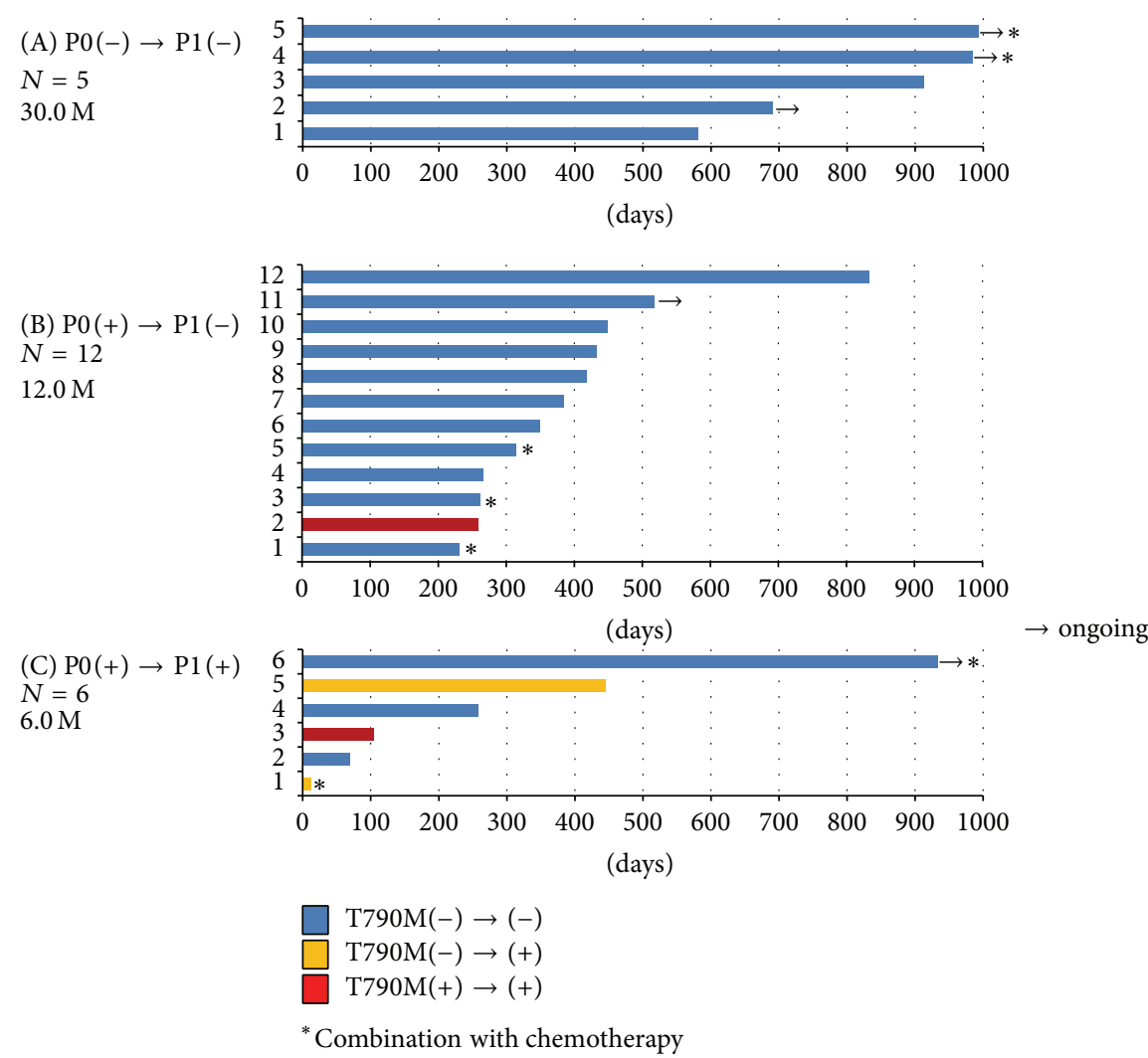

FIGURE 1: Relation between plasma EGFR mutations and duration of EGFR-TKIs treatment. (A): group of cases having no EGFR mutation in the plasma before TKI treatment (P0) and during TKI treatment (P1). (B): group of patients whose EGFR mutation status converted from positive at $\mathrm{P} 0$ to negative at $\mathrm{P} 1$. (C): group of patients with mutations both at $\mathrm{P} 0$ and at P1. Arrows indicate cases that are still under treatment. Asterisks show cases receiving combination of TKIs and chemotherapy.

all identical to the baseline mutations. Patients negative for EGFR mutations had longer PFS, more than 30 months, with no median PFS (Figure 2). Three of five cases in group A were under treatment at cut-off point. Median treatment duration for groups $\mathrm{B}$ and $\mathrm{C}$ was 12 months and 6 months, respectively. There are no serious differences in characteristics among these three groups (Table 3). Because patients negative for plasma mutations tended to have localized tumors, long treatment duration was acceptable. The disappearance of EGFR mutations in the plasma samples of patients after TKI treatment might be a favorable predictive factor for response to EGFR-TKI treatment.

3.4. T790M Detection in the Plasma and Correlation with TKI Efficacy. At baseline, the drug resistance mutation T790M, a de novo mutation, was detected in 2 of 24 cases without the T790M mutation detected by conventional analyses in the tumor (Table 4). These 2 cases had short treatment duration compared with the T790M-negative cases at baseline. Detection of the de novo T790M mutation might be related to the high sensitivity of this analysis. At P1, T790M was newly detected in 2 cases. One case discontinued TKI treatment less than one month after initiation due to pneumotoxicity. The other case, having postoperative recurrence, underwent TKI treatment for more than a year. At disease progression (P2), T790M mutation was detected in 8 of 16 cases (50.0\%) with sufficient frequency, and the activating mutation was

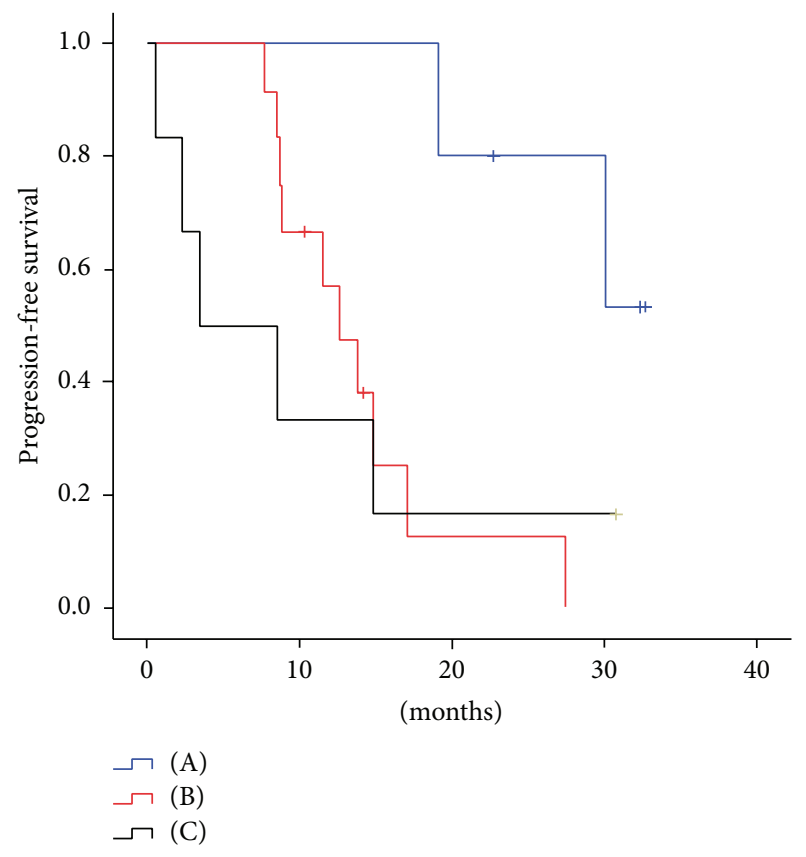

FIGURE 2: Kaplan-Meier curves for progression-free survival of the groups described in Figure 1.

observed in 11 of 16 cases (68.8\%). Only 3 cases who could undergo rebiopsy at $\mathrm{P} 2$ had both the activating mutation and 
TABLE 3: Detection of EGFR mutations from tissue and plasma samples.

\begin{tabular}{|c|c|c|c|c|c|}
\hline \multirow[b]{2}{*}{$N(\%)$} & Tissue & \multicolumn{3}{|c|}{ Plasma } & \multirow{2}{*}{$\begin{array}{c}\text { Tissue } \\
\text { After PD }\end{array}$} \\
\hline & Before treatment & $\begin{array}{c}\text { Before } \\
\text { treatment } \\
\text { P0 (\%) }\end{array}$ & $\begin{array}{l}\text { Under treatment } \\
\qquad \text { P1 (\%) }\end{array}$ & $\begin{array}{l}\text { After PD } \\
\text { P2 (\%) }\end{array}$ & \\
\hline Number of samples & 24 & 24 & 23 & 16 & 3 \\
\hline Activating mutation & 24 & $19(79.2)$ & $6(26.1)$ & $11(68.8)$ & $3(100)$ \\
\hline Drug-resistant mutation (T790M) & NA & $2(8.3)$ & $4(17.4)$ & $8(50.0)$ & $3(100)$ \\
\hline
\end{tabular}

TABLE 4: Characteristics of patients with alteration of the EGFR mutation status after EGFR-TKI treatment.

\begin{tabular}{|c|c|c|c|}
\hline & $\begin{array}{l}\text { Persistent plasma EGFR mutation } \\
\text { negative }(\mathrm{A})\end{array}$ & $\begin{array}{c}\text { Conversion of plasma EGFR } \\
\text { mutation from positive to } \\
\text { negative (B) }\end{array}$ & $\begin{array}{c}\text { Persistent plasma EGFR mutation } \\
\text { positive }(\mathrm{C})\end{array}$ \\
\hline Total, $n$ & 5 & 12 & 6 \\
\hline \multicolumn{4}{|l|}{ Age } \\
\hline Median (range) & $68(55-84)$ & $65.5(46-87)$ & $66.5(58-79)$ \\
\hline \multicolumn{4}{|l|}{ Gender } \\
\hline Female & $5(100)$ & $7(58.3)$ & $3(50.0)$ \\
\hline Male & $0(0.0)$ & $5(41.7)$ & $3(50.0)$ \\
\hline \multicolumn{4}{|l|}{ Smoking status } \\
\hline Never & $4(80.0)$ & $9(75.0)$ & $4(66.6)$ \\
\hline Former & $1(20.0)$ & $1(8.3)$ & $1(16.7)$ \\
\hline Current & $0(0.0)$ & $2(16.7)$ & $1(16.7)$ \\
\hline \multicolumn{4}{|l|}{ ECOG PS } \\
\hline 0 & $3(60.0)$ & $4(33.3)$ & $0(0.0)$ \\
\hline 1 & $2(40.0)$ & $7(58.3)$ & $5(83.3)$ \\
\hline 2 & $0(0.0)$ & $1(8.3)$ & $1(16.7)$ \\
\hline \multicolumn{4}{|l|}{ Stage } \\
\hline IIIA & $1(20.0)$ & $1(8.3)$ & $0(0.0)$ \\
\hline IV & $4(80.0)$ & $11(91.7)$ & $6(100)$ \\
\hline IV-M1a & $4(100)$ & $4(36.4)$ & $0(0.0)$ \\
\hline IV-M1b & $0(0.0)$ & $7(63.6)$ & $6(100)$ \\
\hline \multicolumn{4}{|c|}{ Tumor EGFR mutation status } \\
\hline del19 & $3(60.0)$ & $8(66.7)$ & $3(50.0)$ \\
\hline L858R & $1(20.0)$ & $4(33.3)$ & $3(50.0)$ \\
\hline L861Q & $1(20.0)$ & $0(0.0)$ & $0(0.0)$ \\
\hline
\end{tabular}

the T790M mutation detected in cytohistological as well as plasma samples. There was a complete match between plasma and cytohistological samples.

\section{Discussion}

This study showed that a high detection rate for EGFR mutations in the blood could be achieved by an improved PNALNA PCR clamp method. Results from plasma and cytohistological samples were approximately $80 \%$ concordant. Detection of mutations in the plasma of patients without extrathoracic metastases was harder than in patients with extrathoracic metastases. The disappearance of activating mutations during TKI treatment represents a candidate for new predictive factors for TKI treatment.
NGS or dPCR had attracted attention over the years as possible methods for liquid biopsy. However, these methodologies were expensive and the enormous amount of data obtained from NGS was difficult to manage. On the other hand, the improved PNA-LNA PCR clamp method could achieve high detection rate of EGFR mutations at low costs. Several recent meta-analyses showed $62-65 \%$ of sensitivity and $88-97 \%$ of specificity [13-16]. A clinically useful detection rate is supposed to be more than $80 \%$, and our method approximately reached this value. Original PNA-LNA PCR clamp methods are commercially available in Japan, but their sensitivity is approximately $1 \%$. We improved the sensitivity to $0.1 \%$ by changing primer sites and a thermal cycler. This method has advantages in the cost-benefit balance compared with dPCR and NGS. This PCR analysis costs about \$200-300 
for main activating and resistance mutations of a plasma specimen. Recently, Thress et al. reported comparisons among cobas EGFR mutation test, amplification refractory mutation system (ARMS)-PCR, droplet dPCR, and BEAMing dPCR in liquid biopsy [17]. Both droplet dPCR and BEAMing dPCR had the highest sensitivity in detecting T790M mutation, followed in order by cobas and ARMS-PCR. At the moment, digital platforms could be superior to nondigital platforms in terms of sensitivity, despite some false positive results obtained by digital platforms.

A second important advantage is that EGFR mutations can be detected in the blood. When liquid biopsies will be clinically available, they will be frequently used to avoid rebiopsies. If factors responsible for the inability to detect EGFR mutations will be elucidated, we will proceed to examine rebiopsies when we get negative results in liquid biopsy. In this study, the most critical factor was the site of the disease, restricted to the chest or not; on the basis of the TNM classification, this represents the distinction between M1b and the rest of the diseases. Activating mutations were observed in all patients with extrathoracic metastases, whereas they were observed in just 5 of 10 patients without extrathoracic metastases $(50.0 \%)$. Some papers reported that it was hard to detect EGFR mutations from plasma samples of patients with intrathoracic disease $[13,18]$. The other paper reported no difference among stages of the disease [19]. Since our data were consistent with these results, we have to keep in mind that it is difficult to get adequate results from liquid biopsies in patients with intrathoracic lesions including pulmonary metastases.

Another issue is the relation between the results of liquid biopsy and the effect of EGFR-TKI. The factor influencing PFS was the absence of any EGFR mutation in the plasma before treatment. As we mentioned above, this factor was only associated with patient with intrathoracic diseases. Intrathoracic distribution of disease without extrathoracic metastases might be a predictive factor for long PFS. Another critical factor is the disappearance of activating mutations in the plasma during EGFR-TKI treatment, which could be closely related to the efficacy of EGFR-TKIs. However, persistent existence of the EGFR T790M mutation, the activating mutation, or both in the plasma, despite TKI treatment, was an unfavorable factor related to short PFS. Tseng et al. showed that cases with persistently detectable mutations in the plasma had shorter PFS and OS than cases with undetectable plasma EGFR mutations at baseline and cases with mutations in the plasma disappearing after EGFR-TKI treatment [18]. This previous report demonstrated that cases with undetectable plasma mutations had longer survival than cases where the mutations disappeared, despite no difference in terms of PFS. Here we emphasize that disappearance of activating mutations in the plasma could be a predictive factor for EGFR-TKI efficacy in addition to the absence of plasma mutations at baseline.

This study has several limitations, including the small sample size and the enrollment of patients from a single institute. Now we are expanding our study collecting samples from several institutes using our highly sensitive method and planning to compare this method to digital PCR or NGS.
In conclusion, we were able to effectively detect EGFR activating or drug-resistant mutations in blood samples using an improved PNA-LNA PCR clamp method, which is inexpensive and accessible compared with $\mathrm{dPCR}$ or nextgeneration DNA sequencing.

$\begin{array}{ll}\text { Abbreviations } & \\ \text { ctDNA: } & \text { Circulating tumor DNA } \\ \text { ECOG: } & \text { Eastern Cooperative } \\ & \text { Oncology Group } \\ \text { EDTA: } & \text { Ethylenediaminetetraa- } \\ & \text { cetic acid } \\ \text { EGFR: } & \text { Epidermal growth factor } \\ & \text { receptor } \\ \text { DCR: } & \text { Disease control rate } \\ \text { dPCR: } & \text { Digital PCR } \\ \text { NGS: } & \text { Next-generation } \\ & \text { sequencing } \\ \text { NSCLC: } & \text { Non-small cell lung } \\ & \text { cancer } \\ \text { OS: } & \text { Overall survival } \\ \text { ORR: } & \text { Objective response rate } \\ \text { PFS: } & \text { Progression-free survival } \\ \text { PNA-LNA PCR clamp method: } & \text { Peptide nucleic } \\ & \text { acid-locked nucleic acid } \\ & \text { polymerase chain } \\ \text { PR: } & \text { reaction clamp method } \\ \text { TKIs: } & \text { Partial response } \\ & \text { Performance status } \\ & \text { Tyrosine kinase } \\ & \text { inhibitors. } \\ & \end{array}$

\section{Competing Interests}

Makoto Maemondo received lecture fee from AstraZeneca, Chugai, and Boehringer Ingelheim. The other authors have no competing interests regarding this paper.

\section{References}

[1] M. Maemondo, A. Inoue, K. Kobayashi et al., "Gefitinib or chemotherapy for non-small-cell lung cancer with mutated EGFR," The New England Journal of Medicine, vol. 362, no. 25, pp. 2380-2388, 2010.

[2] T. Mitsudomi, S. Morita, Y. Yatabe et al., "Gefitinib versus cisplatin plus docetaxel in patients with non-small-cell lung cancer harbouring mutations of the epidermal growth factor receptor (WJTOG3405): an open label, randomised phase 3 trial," The Lancet Oncology, vol. 11, no. 2, pp. 121-128, 2010.

[3] C. Zhou, Y.-L. Wu, G. Chen et al., "Erlotinib versus chemotherapy as first-line treatment for patients with advanced EGFR mutation-positive non-small-cell lung cancer (OPTIMAL, CTONG-0802): a multicentre, open-label, randomised, phase 3 study," The Lancet Oncology, vol. 12, no. 8, pp. 735-742, 2011.

[4] R. Rosell, E. Carcereny, R. Gervais et al., "Erlotinib versus standard chemotherapy as first-line treatment for European patients with advanced EGFR mutation-positive non-small-cell lung cancer (EURTAC): a multicentre, open-label, randomised 
phase 3 trial," The Lancet Oncology, vol. 13, no. 3, pp. 239-246, 2012.

[5] L. V. Sequist, J. C.-H. Yang, N. Yamamoto et al., "Phase III study of afatinib or cisplatin plus pemetrexed in patients with metastatic lung adenocarcinoma with EGFR mutations," Journal of Clinical Oncology, vol. 31, no. 27, pp. 3327-3334, 2013.

[6] J. C.-H. Yang, Y.-L. Wu, M. Schuler et al., "Afatinib versus cisplatin-based chemotherapy for EGFR mutation-positive lung adenocarcinoma (LUX-Lung 3 and LUX-Lung 6): analysis of overall survival data from two randomised, phase 3 trials," The Lancet Oncology, vol. 16, no. 2, pp. 141-151, 2015.

[7] H. A. Yu, M. E. Arcila, N. Rekhtman et al., "Analysis of tumor specimens at the time of acquired resistance to EGFRTKI therapy in 155 patients with EGFR-mutant lung cancers," Clinical Cancer Research, vol. 19, no. 8, pp. 2240-2247, 2013.

[8] Z. Li, Y. Zhang, W. Bao, and C. Jiang, "Insufficiency of peripheral blood as a substitute tissue for detecting EGFR mutations in lung cancer: a meta-analysis," Targeted Oncology, vol. 9, no. 4, pp. 381-388, 2014.

[9] Y. Nagai, H. Miyazawa, K. Hagiwara et al., "Genetic heterogeneity of the epidermal growth factor receptor in non-small cell lung cancer cell lines revealed by a rapid and sensitive detection system, the peptide nucleic acid-locked nucleic acid PCR clamp," Cancer Research, vol. 65, no. 16, pp. 7276-7282, 2005.

[10] K. Kobayashi, A. Inoue, K. Usui et al., "First-line gefitinib for patients with advanced non-small-cell lung cancer harboring epidermal growth factor receptor mutations without indication for chemotherapy," Journal of Clinical Oncology, vol. 27, no. 9, pp. 1394-1400, 2009.

[11] M. Maemondo, Y. Minegishi, A. Inoue et al., "First-line gefitinib in patients aged 75 or older with advanced non-small cell lung cancer harboring epidermal growth factor receptor mutations: NEJ 003 study," Journal of Thoracic Oncology, vol. 7, no. 9, pp. 1417-1422, 2012.

[12] S. Sugawara, S. Oizumi, K. Minato et al., "Randomized phase II study of concurrent versus sequential alternating gefitinib and chemotherapy in previously untreated non-small cell lung cancer with sensitive EGFR mutations: NEJ005/TCOG0902," Annals of Oncology, vol. 26, no. 5, pp. 888-894, 2015.

[13] M. Qiu, J. Wang, Y. Xu et al., "Circulating tumor DNA is effective for the detection of EGFR mutation in non-small cell lung cancer: a meta-analysis," Cancer Epidemiology Biomarkers and Prevention, vol. 24, no. 1, pp. 206-212, 2015.

[14] Y. Wu, H. Liu, X. Shi, and Y. Song, "Can EGFR mutations in plasma or serum be predictive markers of non-small-cell lung cancer? A meta-analysis," Lung Cancer, vol. 88, no. 3, pp. 246253, 2015.

[15] J.-Y. Douillard, G. Ostoros, M. Cobo et al., "Gefitinib treatment in EGFR mutated caucasian NSCLC: circulating-free tumor DNA as a surrogate for determination of EGFR status," Journal of Thoracic Oncology, vol. 9, no. 9, pp. 1345-1353, 2014.

[16] C. Mao, J.-Q. Yuan, Z.-Y. Yang, X.-H. Fu, X.-Y. Wu, and J.-L. Tang, "Blood as a substitute for tumor tissue in detecting EGFR mutations for guiding EGFR TKIs treatment of nonsmall cell lung cancer," Medicine, vol. 94, no. 21, article e775, 2015.

[17] K. S. Thress, R. Brant, T. H. Carr et al., "EGFR mutation detection in ctDNA from NSCLC patient plasma: a crossplatform comparison of leading technologies to support the clinical development of AZD9291," Lung Cancer, vol. 90, no. 3, pp. 509-515, 2015.
[18] J.-S. Tseng, T.-Y. Yang, C.-R. Tsai et al., "Dynamic plasma EGFR mutation status as a predictor of EGFR-TKI efficacy in patients with EGFR-mutant lung adenocarcinoma," Journal of Thoracic Oncology, vol. 10, no. 4, pp. 603-610, 2015.

[19] H.-R. Kim, S. Y. Lee, D.-S. Hyun et al., "Detection of EGFR mutations in circulating free DNA by PNA-mediated PCR clamping," Journal of Experimental and Clinical Cancer Research, vol. 32, no. 1, article 50, 2013. 


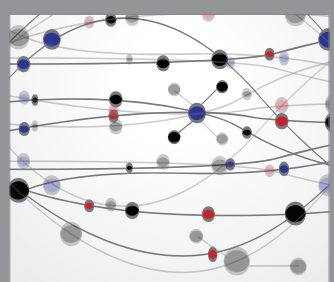

The Scientific World Journal
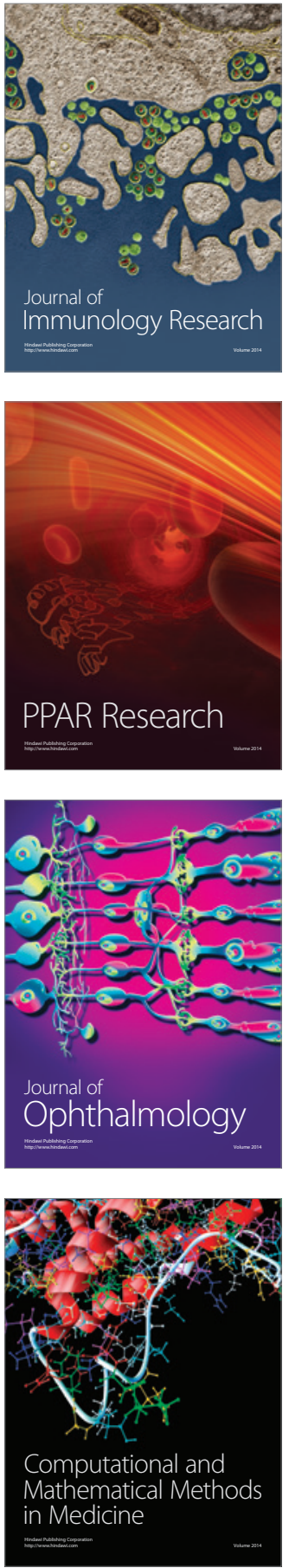

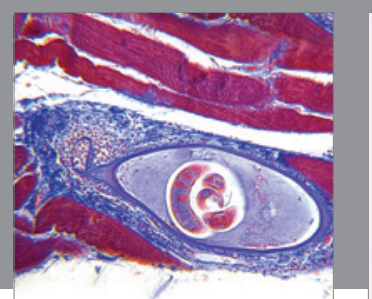

Gastroenterology Research and Practice

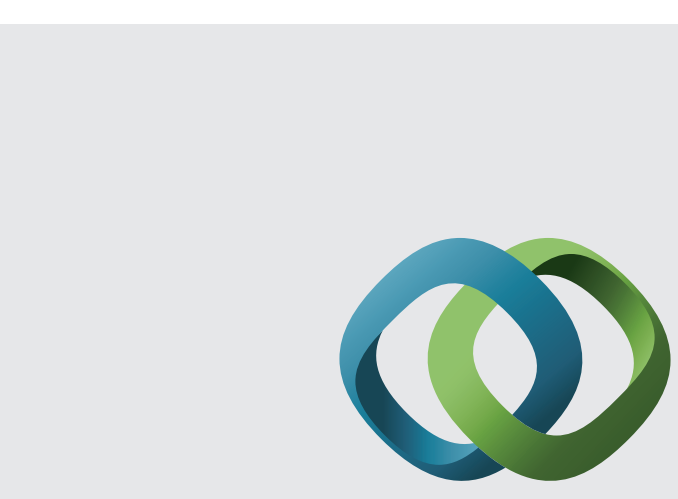

\section{Hindawi}

Submit your manuscripts at

http://www.hindawi.com
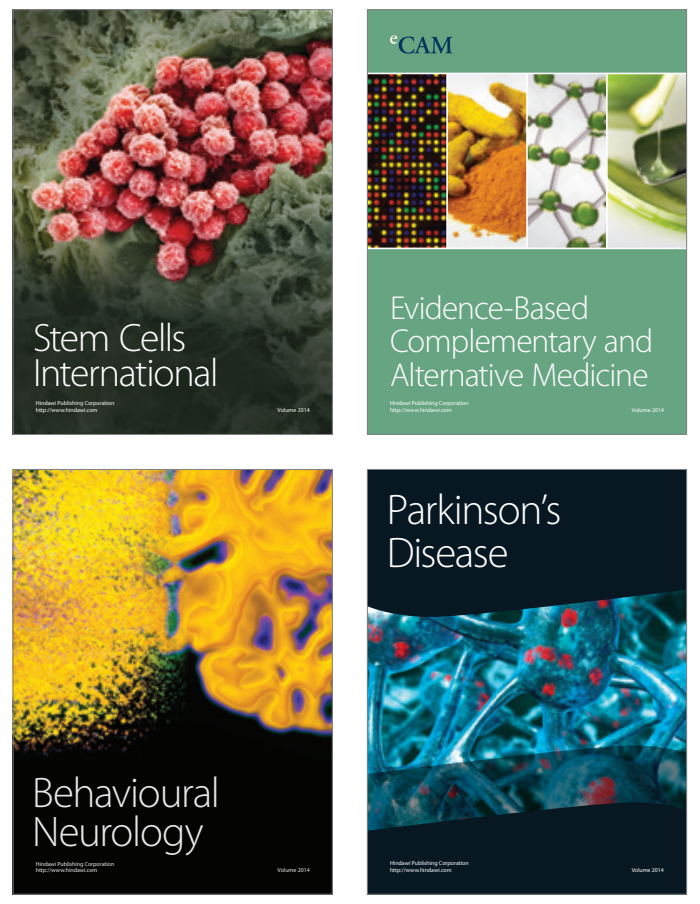
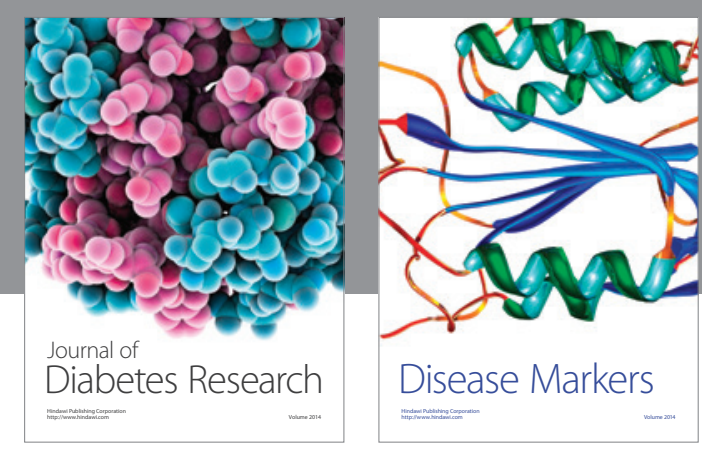

Disease Markers
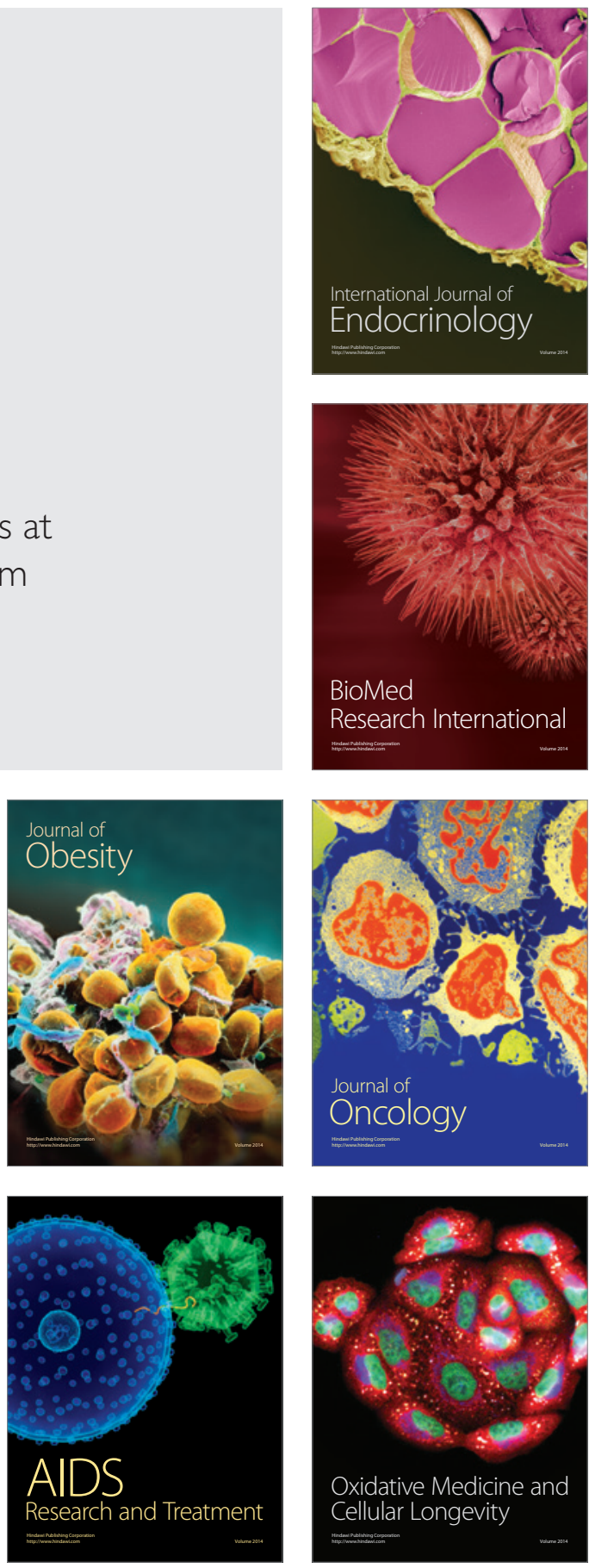